\title{
What Does 3D Echocardiography Add to 2D Echocardiography in the Assessment of Mitral Regurgitation?
}

\author{
Tadafumi Sugimoto $^{1,2} \cdot$ Raluca Dulgheru ${ }^{1,2}$ - Stella Marchetta ${ }^{1,2}$ - Federica Ilardi ${ }^{1,2}$. \\ Laura Contu $^{1,2}$ - Yun Yun Go ${ }^{1,2}$ - Patrizio Lancellotti ${ }^{1,2,3}$
}

(C) Springer Science+Business Media, LLC 2017

\begin{abstract}
Purpose of Review The purpose of this review was to elucidate the additional value of $3 \mathrm{D}$ echocardiography for the assessment of mitral regurgitation (MR) compared to standard 2D echocardiography.

Recent Findings 3D echocardiography provides key information, aetiology, degenerative mitral valve disease vs. secondary MR, causes and mechanism, severity by measurements of effective regurgitant orifice area and regurgitant volume; likelihood of reparability and assessment of pre- and intra-mitral valve transcatheter procedures.

Summary 3D echocardiography as a promising method for assessment of MR is useful and crucial for research, clinical practice and patient management in all heart valve team members.
\end{abstract}

Keywords 3D echocardiography $\cdot$ Assessment $\cdot$ Mitral regurgitation · Transoesophageal echocardiography ·

Transcatheter procedures

\section{Abbreviations \\ MR Mitral regurgitation \\ 2D 2-Dimensional}

This article is part of the Topical Collection on Echocardiography

Patrizio Lancellotti

plancellotti@chu.ulg.ac.be

1 Department of Cardiology, University Hospital Sart Tilman, B 4000 Liege, Belgium

2 GIGA Cardiovascular Science, Heart Valve Clinic, Imaging Cardiology, University of Liège Hospital, Liège, Belgium

3 Gruppo Villa Maria Care and Research, Anthea Hospital, Bari, Italy
3D 3-Dimensional

DMVD Degenerative mitral valve disease

LV Left ventricular

PISA Proximal isovelocity surface area

EROA Effective regurgitant orifice area

\section{Introduction}

Mitral regurgitation (MR) is the second most frequent heart valve disease in developed countries. The assessment of the aetiology, mechanism, severity and reparability is essential for the decision-making process for transcatheter/surgical MR treatment. Transthoracic echocardiography is recommended as the first-line imaging modality for assessment of mitral valve anatomy, MR severity, its haemodynamics and ventricular consequences $[1,2 \bullet \bullet]$. When transthoracic echocardiography is of non-diagnostic value or whenever further diagnostic refinement is required, transoesophageal echocardiography is advocated. Although 2D echocardiography is the primary imaging modality in both transthoracic and transoesophageal echocardiography, this paper focuses on the additional value of 3D echocardiography in the assessment of MR.

\section{Aetiology}

MR is classified as primary (organic) or secondary (functional) [1]. Common causes of primary MR are degenerative mitral valve disease, rheumatic disease and endocarditis. Degenerative mitral valve disease, including diffuse myxomatous degeneration, fibroelastic degeneration, Marfan, Ehlers-Danlos syndrome and annular calcification, is often morphologically sub-classified as the following: (1) billowing (a part of the mitral valve body protrudes into the left atrium but coaptation 
is preserved beyond the annular plane), (2) floppy (a morphologic abnormality with thickened leaflet), (3) prolapse (a part of coaptation line is behind the annular plane), (4) flail (a free edge of a leaflet is completely reversed in the left atrium) [1]. The most common phenotype of mitral valve prolapse is diffuse myxomatous degeneration. Mitral valve annular dilatation in mitral valve prolapse is associated with the basal predominance of left ventricular (LV) dilatation and attenuated contraction, which can be altered by surgical mitral valvuloplasty with annulus reduction [3・•]. A recent study using real-time 3D transoesophageal echocardiography has shown that flattening of the annular saddle shape is associated with progressive leaflet billowing and increased frequencies of chordal rupture in mitral valve prolapse [4].

Common causes of secondary MR are ischaemic heart disease and dilated cardiomyopathy. It results from an imbalance between tethering forces (annular dilatation, LV dilatation, papillary muscles displacement, LV sphericity, high left atrial pressure) and closing forces (reduction of LV contractility, global LV dyssynchrony, papillary muscle dyssynchrony, altered mitral systolic annular contraction) [1]. 3D echocardiography has contributed most to the understanding of the structural remodelling that accompanies secondary MR [5-7] and has demonstrated superiority over 2D echocardiography in measuring LV volumes, localizing and assessing the extent of mitral valve deformation, and determining the shape of the regurgitant orifice [8] (Fig. 1).

\section{Mechanism}

The rationale for mitral valve treatment depends on the MR mechanism, and therefore it is essential to identify and understand the pathophysiology of MR. The Carpentier's classification of mitral leaflet pathology is defined surgically as follows: (1) type I, normal leaflet motion (annular dilation, leaflet perforation); (2) type II, excessive leaflet motion (leaflet prolapsed, chordal rupture, chordal elongation, papillary muscle rupture, papillary muscle elongation); (3) type III, restricted leaflet motion (type IIIa, during diastole and systole due to shortening of the chordae and/or leaflet thickening such as in rheumatic MR and type IIIb, during systole such as in ischaemic MR).

3D dynamic imaging has demonstrated the dynamic nature of mitral annulus physiology during the cardiac cycle. In both healthy subjects and myxomatous valve patients, annular area increased between early and middle or late systole. In this period, annular area returns to diastolic area in the normal annulus but enlarges beyond diastolic area in myxomatous valve patients. Then, saddle-shape deepening occurs later and is less pronounced in myxomatous valve patients compared with healthy subjects [9]. Moreover, a recent study using real-time $3 \mathrm{D}$ transoesophageal echocardiography has demonstrated that the phenotypes of degenerative mitral valve disease (fibroelastic degeneration vs. diffuse myxomatous degeneration) display considerably different valvular pathophysiology $\left[10^{\bullet} \cdot\right.$. In fibroelastic degeneration, annular dynamics is similar to that in healthy subjects (early systolic anteroposterior contraction and saddle-shape accentuation with stable intercommissural diameter). In diffuse myxomatous degeneration, however, annular dynamics is greatly abnormal in early systole (reduced anteroposterior contraction and saddle-shape accentuation) and late systole (abnormal enlargement because of marked intercommissural enlargement).

Patient-specific mitral leaflet strain analysis using 3D transoesophageal echocardiography has reported that patients with primary MR have higher mitral valve strain compared to normal subjects, and that strain concentration is the highest in the commissural zone, intermediate in the annulus and coaptation zones and the lowest in the central zone [11••]. Recently, the relationship between annular dilatation caused by atrial fibrillation and MR has been also studied by 3D transoesophageal echocardiography $[12 \bullet \bullet]$. Although annular area is greater in atrial fibrillation patients both with and without MR than healthy subjects, total leaflet area remains greater in atrial fibrillation patients without MR compared to those with MR or healthy subjects.

Secondary MR develops because of a combination of mitral leaflet tethering secondary to LV dilatation/deformation with papillary displacement/discoordination, annular dilatation/dysfunction, insufficient LV-generated closing forces attributable to reduction of LV contractility and global LV/ papillary muscle dyssynchrony. Tethering of the mitral leaflets is the principal lesion of secondary MR and results in restriction of systolic leaflet motion (type IIIb of Carpentier's classification). Measures of mitral valve configuration parameters are now readily available using 3D echocardiography, which reduces the risk of foreshortened views and off-axis planes by $2 \mathrm{D}$ echo. It provides accurate evaluation of leaflets remodelling and mitral annulus dimensions and dynamics. Tenting volume shape can be reconstructed through offline analysis from 3D-acquired data sets. Examining the tenting volume shape makes it easy to identify the pattern of tethering (symmetric vs. asymmetric) on the MV leaflets, asymmetric tethering exhibiting a tenting volume deformed toward the most tethered segment of the leaflet and pointing out the part of the leaflet that is predominantly stretched. The tenting volume, the volume enclosed between the MV leaflets and the annular plane in mid-systole, is closely related to MR severity and is a reliable marker of tethering severity $[13,14]$.

\section{MR Severity}

2D echocardiography plays an essential role in the assessment of MR severity. The criteria for grading MR with 2D 
Fig. 1 Dynamicity of the regurgitant orifice during systole as depicted by $3 \mathrm{D}$ transthoracic echocardiography

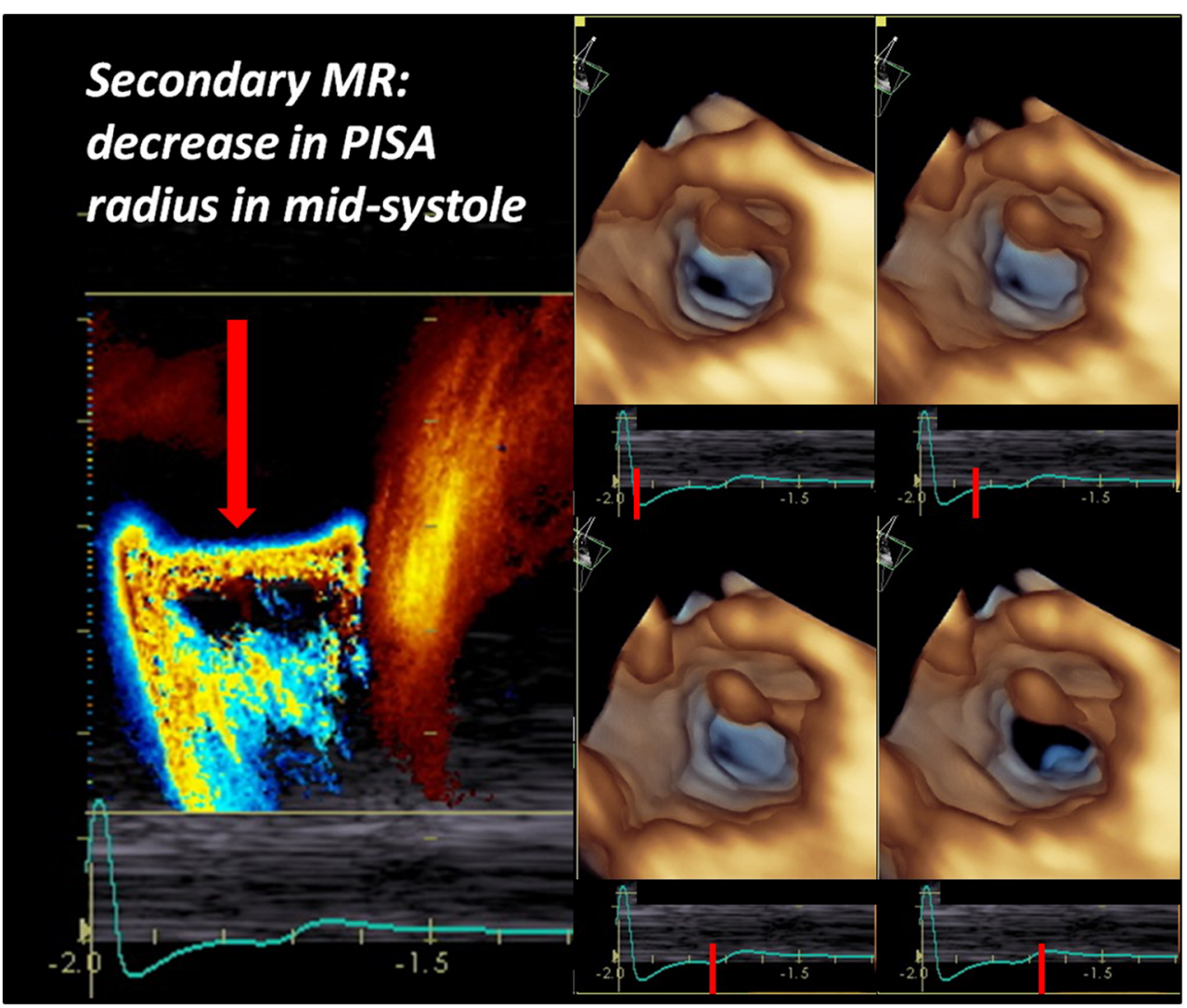

echocardiography are described in Table $1[15,16 \bullet]$. Qualitatively, colour flow imaging is the most common way to assess MR severity. The density of the continuous wave Doppler envelope of the MR jet can provide clues about MR degree. The presence of a flow convergence at a Nyquist limit of $50-60 \mathrm{~cm} / \mathrm{s}$ should alert to the presence of significant MR. Semiquantitatively, a vena contracta $<3 \mathrm{~mm}$ indicates mild MR whereas a width $\geq 7 \mathrm{~mm}$ defines severe MR. Pulsed Doppler evaluation of pulmonary venous flow pattern is also an aid to assess MR severity. A peak E velocity $>1.5 \mathrm{~m} / \mathrm{s}$ suggests severe MR in the absence of mitral stenosis whereas a dominant A wave basically excludes severe MR. The pulsed Doppler mitral to aortic time velocity integral ratio (i.e. $>1.4$ ) can be used as an index in primary severe MR. Quantitatively, the flow convergence method is the most recommended quantitative approach whenever feasible. The radius of the proximal isovelocity surface area (PISA) is measured at mid-systole using the first aliasing. The PISA method is based on the assumption of haemispheric symmetry of the velocity distribution proximal to the regurgitant lesion, which may not hold for eccentric jets, multiple jets and complex or elliptical regurgitant orifices. In the European Society of Cardiology (ESC)/European Association for Cardio-Thoracic Surgery (EACTS) guidelines [15], primary MR is considered severe if effective regurgitant orifice area (EROA) is $\geq 40 \mathrm{~mm}^{2}$ and regurgitant volume $\geq 60 \mathrm{~mL}$. In secondary $\mathrm{MR}$, the thresholds of severity are $20 \mathrm{~mm}^{2}$ and $30 \mathrm{~mL}$, respectively. In the 2017 American Heart Association (AHA)/American College of Cardiology (ACC) focused update guidelines [16•], on the basis of the criteria used for determination of severe MR in randomized controlled trials of surgical intervention, both primary and secondary MR are considered severe if EROA is $\geq 40 \mathrm{~mm}^{2}$, regurgitant volume $\geq 60 \mathrm{~mL}$ and regurgitant fraction $\geq 50 \%$. The presence of severe MR has significant haemodynamic effects, primarily on the LV and left atrium. When MR is more than mild, providing the LV diameters, volumes and ejection fraction as well as the left atrium volume and the pulmonary arterial systolic pressure in the final echocardiographic report is mandatory. 3D echo has demonstrated superiority over $2 \mathrm{D}$ echo and similar accuracy to cardiac magnetic resonance imaging in evaluating $\mathrm{LV}$ volumes and LV ejection fraction [17].

Assessment of MR using 3D echocardiography is reasonable to provide additional information on severity, especially in measurements of EROA and regurgitant volume. In patients with primary or secondary MR, 3D EROA is well correlated with cardiac magnetic resonance imaging regurgitant volume [18]. 3D echocardiography overcomes the current limitations of $2 \mathrm{D}$ echo by a direct measurement of vena contracta area (direct planimetry) in the plane perpendicular to the direction of the regurgitant jet with no geometric assumptions. 3Dderived vena contracta area has been shown to correlate more 


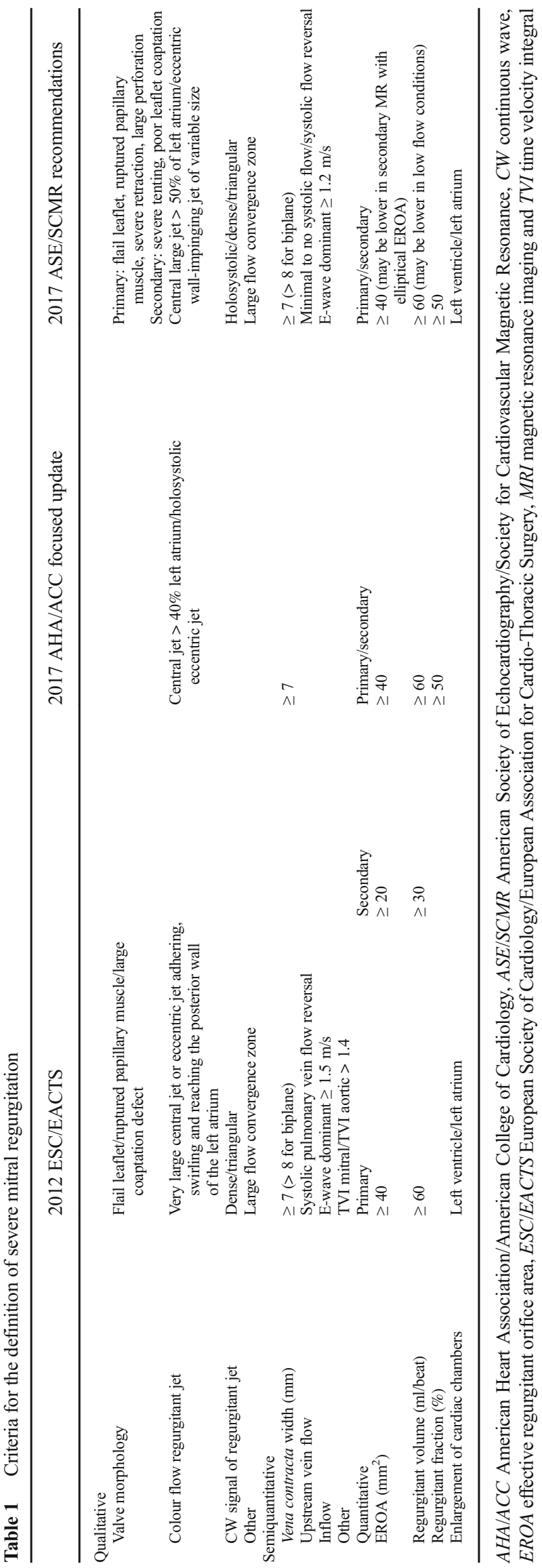

closely with Doppler-derived EROA than $2 \mathrm{D}$ vena contracta diameter. A vena contracta area of $\geq 0.41 \mathrm{~cm}^{2}$ seems to be indicative of severe MR although further validation of this cut-off is necessary given that the comparison standard in most have been conventional 2D estimates of severity of MR [19]. The PISA method, as assessed by 2D echocardiography, assumes that the flow convergence area is haemispherical. As demonstrated by 3D echocardiography, flow convergence area is frequently haemielliptic in secondary MR (Fig. 2) and applying PISA radius method in this case might lead to underestimation of EROA and regurgitant volume. A recent research demonstrated that the 3D PISA method is more accurate than the current the 2D PISA method in these patients [20••]. With 3D echocardiography, it is also possible to directly measure the 3D surface of proximal isovelocity layers without raw geometric assumptions [21]. This approach is an accurately correlated cardiac magnetic imaging with cardiac magnetic resonance imaging regurgitant volume in secondary MR, although further confirmation of this is required in larger numbers and in multiple centres [18]. However, it is not clear whether these $3 \mathrm{D}$ echo measurements provide incremental information to standard 2D echo measures for predicting outcome after treatment.

\section{Reparability}

In the decision-making process for transcatheter/surgical MR treatment, a heart valve team should consider various factors affecting the individual risk: symptoms, severity of MR, haemodynamic effects of MR, operative risk and feasibility of repair. There is a heterogeneous rate of mitral valve repair among hospitals and among surgeons [22], although mortality and complications associated with mitral valve repair has been reported to be lower than with mitral valve replacement. Although 2D echo has been established as the reference method for mitral valve analysis, 3D transthoracic and transoesophageal echocardiography add significant information on, especially in patients with complex lesions. Unlike 2D techniques, which require multiple tomographic views to examine the different parts of the mitral valve leaflets, real-time 3D echocardiography provides a comprehensive assessment of the mitral valve in only one view, the "en face" view from the left atrium (surgeon's view, Fig. 3) or the LV, leading to more effective communication with cardiac surgeons [23]. Several studies have demonstrated, at the time of surgery (surgical inspection), the superiority of 3D over 2D echocardiography [24, 25]. 3D transthoracic echocardiography has been shown to be feasible and accurate in identifying the location of MV prolapse with sensitivity comparable to $2 \mathrm{D}$ transoesophageal echocardiography when the quality of images is sufficient [26]. Characteristics of 2D and 3D-derived echocardiographic parameters obtainable in MR are described in Table 2. 
Fig. 2 Elliptical regurgitant orifices assessed by $2 \mathrm{D}$ and 3D transthoracic echocardiography

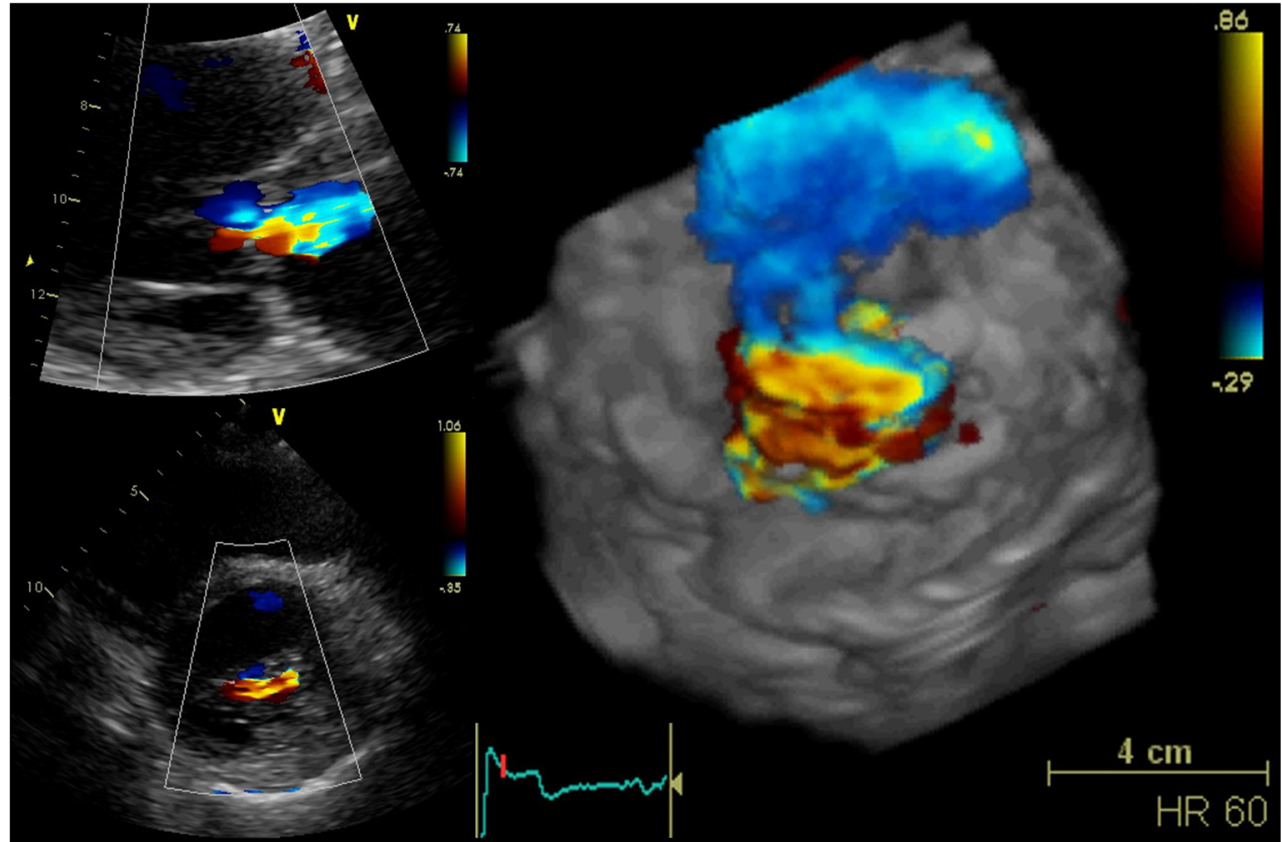

Isolated cleft of the anterior mitral valve leaflet is a rare but well-known finding. During surgical inspection when the heart is collapsed, it is sometimes difficult to identify cleft and cleft-like indentations, especially if the scallops are diseased. 2D transoesophageal echocardiography, despite multiplane imaging, often has difficulty identifying typical cleft and cleft-like indentations, which may be associated with MR and failure of mitral valve repair. 3D transoesophageal echocardiography with proper "en face" image display from the ventricular side has high sensitivity, specificity, positive predictive value and negative predictive value for cleft-like indentation diagnosis $[27 \bullet \bullet]$. Detection of the mitral valve dysfunction following mitral valve repair is also required. 2D and 3D transoesophageal echocardiography should be performed in all cases of recurrent mitral valve dysfunction because of superior spatial resolution and anatomic detail [28•].

\section{MR Assessment for Transcatheter Procedures}

During the past few years, several percutaneous transcatheter mitral valve repair technologies have emerged as possible
Fig. 3 Surgeon's view of the mitral valve $\mathrm{P} 2$ prolapse by $3 \mathrm{D}$ transoesophageal echocardiography

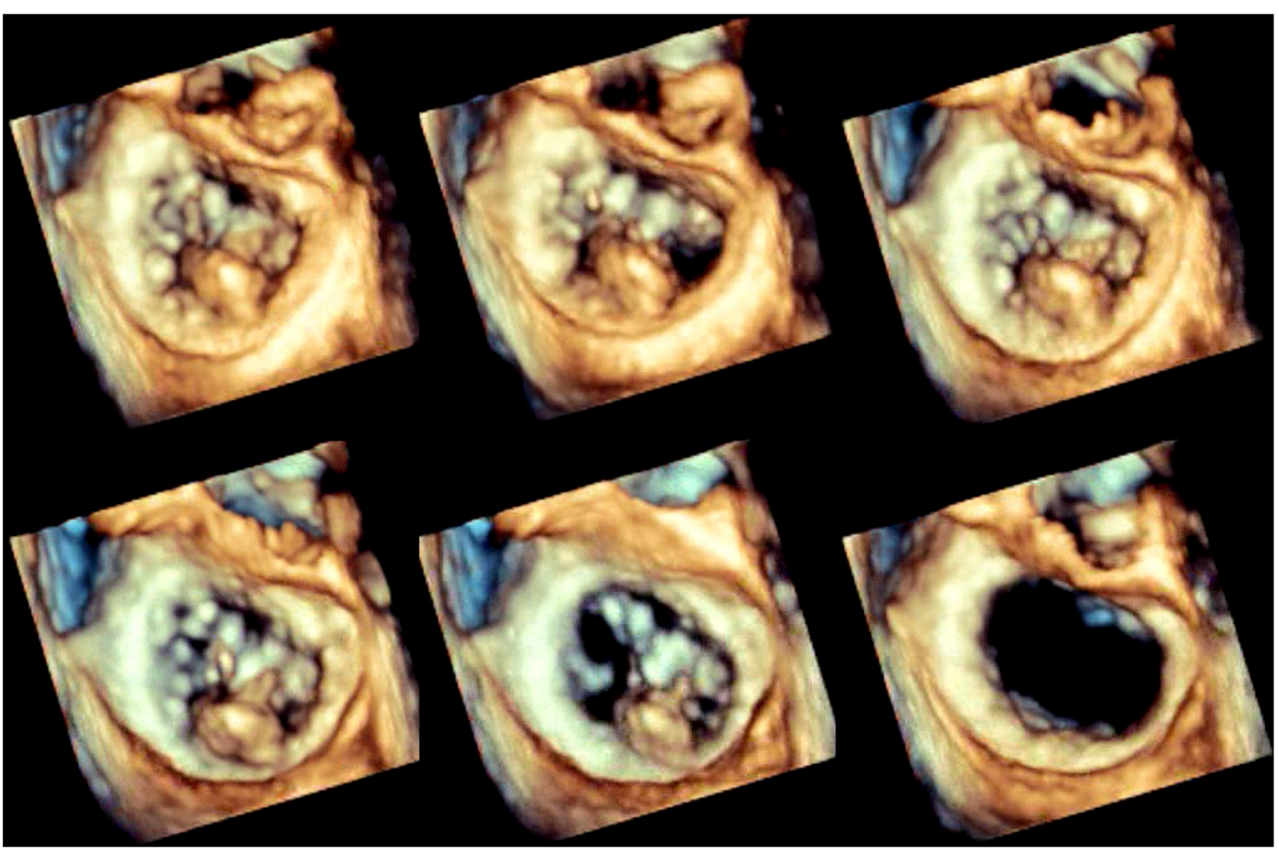


Table 2 2D and 3D-derived echocardiographic parameters obtainable in MR

\begin{tabular}{|c|c|c|}
\hline Mitral parameters & 2D TOE & $3 \mathrm{D}$ TOE \\
\hline \multicolumn{3}{|l|}{ Annulus } \\
\hline Intercommissural distance & + & ++ \\
\hline Septo-lateral distance & + & ++ \\
\hline Perimeter & + & ++ \\
\hline Annulus height & - & ++ \\
\hline Annulus dynamics & - & ++ \\
\hline \multicolumn{3}{|l|}{ Leaflets } \\
\hline Anterior leaflet area & - & ++ \\
\hline Posterior leaflet area & - & ++ \\
\hline Posterior leaflet angle & + & ++ \\
\hline Anterior leaflet angle & + & ++ \\
\hline Coaptation depth & + & ++ \\
\hline Coaptation indexes & - & ++ \\
\hline Leaflet coaptation area & - & ++ \\
\hline Tenting area & + & ++ \\
\hline Tenting volume & - & ++ \\
\hline \multicolumn{3}{|l|}{ Interpapillary distance: } \\
\hline Papillary muscle tip & + & + \\
\hline Papillary muscle body & + & + \\
\hline \multicolumn{3}{|l|}{ Ventricle } \\
\hline LV end-diastolic volume & + & ++ \\
\hline LV end-systolic volume & + & ++ \\
\hline LV dyssynchrony (global/PMs) & + & ++ \\
\hline Vena contracta shape & + & +++ \\
\hline PISA shape & + & +++ \\
\hline
\end{tabular}

$L V$ left ventricle, $P I S A$ proximal isovelocity surface area, $P P M$ papillary muscle, TOE transoesophageal echocardiography

alternatives to open surgery for high-risk patients. As new therapeutic targets, leaflets (percutaneous leaflet plication, leaflet coaptation, leaflet ablation), annulus (indirect annuloplasty through the coronary sinus or direct annuloplasty), chordae (percutaneous chordal implantation) and LV (percutaneous LV remodelling) are proposed and still under development except for the leaflet approaches with the use of MitraClip (edge-to-edge repair) [29•]. MitraClip therapy has proven successful in reducing the severity of MR and in improving the LV dimension, patient's symptoms, quality-of-life and functional capacity [30]. Transcatheter mitral valve replacement is also recognized as a new therapeutic procedure and may have the potential to become an alternative to treat severe MR in patients with high surgical risk. For transcatheter mitral valve replacement, 3D transoesophageal echocardiography is most useful for anatomical assessment and quantification, annular segmentation (aortic cusps, aortomitral continuity, trigone, left atrial appendage, posterior annulus) and landing zone characteristics (annular calcium, mitral valve prolapse, mitral annular disjunction, myocardial shelf, leaflet length, directly inserting papillary muscles), and for intraprocedural guidance by providing an en face view $[31 \bullet \cdot, 32 \bullet$. For paravalvular percutaneous closure, 3D transoeso-phageal echocardiography can be used intraprocedurally and also permits assessment of location and severity of paravalvular leak.

\section{Conclusion}

3D echocardiography provides additional value for the assessment of MR. Aetiologically, 3D echocardiography has contributed to the understanding of the structural remodelling and the interrelationship between the mitral valve and LV in both primary and secondary MR. The assessment of the dynamic nature of mitral annulus physiology and pathophysiology in MR by real-time 3D transoesophageal echocardiography gives new insights into the mechanisms of MR. From a clinical point of view, the application of assessment of MR severity using 3D echocardiography is still challenging as a standard method. Although there is less evidence to show the therapeutic effect of 3D echocardiography-guided mitral valve repair, preand intra-operative 3D "en face" images help heart valve team members communicate with each other. For transcatheter mitral valve procedures, 3D transoesophageal echocardiography is essential for pre- and intra-procedural assessment such as annular segmentation and landing zone characteristics and for intraprocedural guidance.

\section{Compliance with Ethical Standards}

Conflict of Interest Tadafumi Sugimoto, Raluca Dulgheru, Stella Marchetta, Federica Ilardi, Laura Contu Yun Yun Go and Patrizio Lancellotti declare that they have no conflict of interest.

Human and Animal Rights and Informed Consent This article does not contain any studies with human or animal subjects performed by any of the authors.

\section{References}

Papers of particular interest, published recently, have been highlighted as:

- Of importance

•- Of major importance

1. Lancellotti P, Tribouilloy C, Hagendorff A, Popescu BA, Edvardsen T, Pierard LA, et al. Scientific Document Committee of the European Association of Cardiovascular Imaging. Recommendations for the echocardiographic assessment of native valvular regurgitation: an executive summary from the European Association of Cardiovascular Imaging. Eur Heart J Cardiovasc Imaging. 2013;14:611-44. 
2.• Zoghbi WA, Adams D, Bonow RO, Enriquez-Sarano M, Foster E, Grayburn PA, et al. Recommendations for noninvasive evaluation of native Valvular regurgitation: a report from the American Society of Echocardiography developed in collaboration with the Society for Cardiovascular Magnetic Resonance. J Am Soc Echocardiogr. 2017;30:303-71. New recommendations for native valvular regurgitation.

3.•• Fukuda S, Song JK, Mahara K, et al. Basal left ventricular dilatation and reduced contraction in patients with mitral valve prolapse can be secondary to annular dilatation: preoperative and postoperative speckle-tracking echocardiographic study on left ventricle and mitral valve annulus interaction. Circ Cardiovasc Imaging. 2016;9. This study investigated relationships between basal predominance of LV dilatation with reduced contraction and MV annular dilatation in patients with mitral valve prolapse.

4. Lee AP, Hsiung MC, Salgo IS, et al. Quantitative analysis of mitral valve morphology in mitral valve prolapse with real-time 3-dimensional echocardiography: importance of annular saddle shape in the pathogenesis of mitral regurgitation. Circulation. 2013;127:832-41.

5. Fattouch K, Castrovinci S, Murana G, et al. Multiplane twodimensional versus real time three-dimensional transesophageal echocardiography in ischemic mitral regurgitation. Echocardiography. 2011;28:1125-32.

6. Watanabe N, Ogasawara Y, Yamaura Y, et al. Quantitation of mitral valve tenting in ischemic mitral regurgitation by transthoracic realtime three-dimensional echocardiography. J Am Coll Cardiol. 2005;45:763-9.

7. Watanabe N, Ogasawara Y, Yamaura Y, et al. Mitral annulus flattens in ischemic mitral regurgitation: geometric differences between inferior and anterior myocardial infarction: a real-time 3-dimensional echocardiographic study. Circulation. 2005;112:I458-62.

8. Ypenburg C, Lancellotti P, Tops LF, et al. Acute effects of initiation and withdrawal of cardiac resynchronization therapy on papillary muscle dyssynchrony and mitral regurgitation. J Am Coll Cardiol. 2007;50:2071-7.

9. Grewal J, Suri R, Mankad S, et al. Mitral annular dynamics in myxomatous valve disease: new insights with real-time 3-dimensional echocardiography. Circulation. 2010;121:1423-31.

10.• Clavel MA, Mantovani F, Malouf J, et al. Dynamic phenotypes of degenerative myxomatous mitral valve disease: quantitative 3dimensional echocardiographic study. Circ Cardiovasc Imaging. 2015;8. This study showed that, for similar MR severity, annular and valvular dynamics are profoundly different in fibroelastic degeneration and diffuse myxomatous degeneration.

11.• Ben Zekry S, Freeman J, Jajoo A, et al. Patient-specific quantitation of mitral valve strain by computer analysis of threedimensional echocardiography: a pilot study. Circ Cardiovasc Imaging. 2016;9. This study shown that patients with organic MR have significant alterations in the intensity of global strain and its regional distribution compared to normal patient population.

12.• Kagiyama N, Hayashida A, Toki M, et al. Insufficient leaflet remodeling in patients with atrial fibrillation: association with the severity of mitral regurgitation. Circ Cardiovasc Imaging. 2017;10. This study showed that leaflet remodeling plays a crucial role in the occurrence of MR in patients with AF.

13. Levack MM, Jassar AS, Shang EK, et al. Three-dimensional echocardiographic analysis of mitral annular dynamics: implication for annuloplasty selection. Circulation. 2012;126:S183-8.

14. Vergnat M, Jassar AS, Jackson BM, et al. Ischemic mitral regurgitation: a quantitative three-dimensional echocardiographic analysis. Ann Thorac Surg. 2011;91:157-64.

15. Joint Task Force on the Management of Valvular Heart Disease of the European Society of Cardiology (ESC), European Association for Cardio-Thoracic Surgery (EACTS), Vahanian A, et al.
Guidelines on the management of valvular heart disease (version 2012). Eur Heart J. 2012;33:2451-96.

16. Nishimura RA, Otto CM, Bonow RO, et al. AHA/ACC focused update of the $2014 \mathrm{AHA} / \mathrm{ACC}$ guideline for the management of patients with valvular heart disease: a report of the American College of Cardiology/American Heart Association task force on clinical practice guidelines. J Am Coll Cardiol. 2017;70:252-89. In this update, cutoff value of EROA and regurgitant volume for severe secondary MR is changed.

17. Dorosz JL, Lezotte DC, Weitzenkamp DA, Allen LA, Salcedo EE. Performance of 3-dimensional echocardiography in measuring left ventricular volumes and ejection fraction: a systematic review and meta-analysis. J Am Coll Cardiol. 2012;59:1799-808.

18. Thavendiranathan P, Liu S, Datta S, et al. Quantification of chronic functional mitral regurgitation by automated 3-dimensional peak and integrated proximal isovelocity surface area and stroke volume techniques using real-time 3-dimensional volume color Doppler echocardiography: in vitro and clinical validation. Circ Cardiovasc Imaging. 2013;6:125-33.

19. Thavendiranathan P, Phelan D, Thomas JD, et al. Quantitative assessment of mitral regurgitation: validation of new methods. J Am Coll Cardiol. 2012;60:1470-83.

20.• Choi J, Heo R, Hong GR, et al. Differential effect of 3-dimensional color Doppler echocardiography for the quantification of mitral regurgitation according to the severity and characteristics. Circ Cardiovasc Imaging. 2014;7:535-44. This study investigated the differential effect of 3D-color Doppler echocardiography in a wide spectrum of MR phenotypes.

21. Grady L, Datta S, Kutter O, et al. Regurgitation quantification using 3D PISA in volume echocardiography. Med Image Comput Comput Assist Interv. 2011;14:512-9.

22. McCarthy PM. Three-dimensional echocardiography is not essential for intraoperative assessment of mitral regurgitation. Circulation. 2013;128:653-8.

23. Tsang W, Lang RM. Three-dimensional echocardiography is essential for intraoperative assessment of mitral regurgitation. Circulation. 2013;128:643-52.

24. Manda J, Kesanolla SK, Hsuing MC, et al. Comparison of real time two-dimensional with live/real time three-dimensional transesophageal echocardiography in the evaluation of mitral valve prolapse and chordae rupture. Echocardiography. 2008;25:1131-7.

25. Ben Zekry S, Nagueh SF, Little SH. Comparative accuracy of two- and three-dimensional transthoracic and transesophageal echocardiography in identifying mitral valve pathology in patients undergoing mitral valve repair: initial observations. J Am Soc Echocardiogr. 2011;24:1079-85.

26. Macnab A, Jenkins NP, Bridgewater BJ, et al. Three-dimensional echocardiography is superior to multiplane transoesophageal echo in the assessment of regurgitant mitral valve morphology. Eur J Echocardiogr. 2004;5:212-22.

27.• Mantovani F, Clavel MA, Vatury O, et al. Cleft-like indentations in myxomatous mitral valves by three-dimensional echocardiographic imaging. Heart. 2015;101:1111-7. This study defined accuracy of cleft-like indentations preoperative diagnosis and 3D myxomatous mitral valve disease morphological features.

28. Spoon JN, Nkomo VT, Suri RM, et al. Mechanisms of mitral valve dysfunction following mitral valve repair for degenerative disease. JACC Cardiovasc Imaging. 2015;8:1223-7. An echocardiography-based imaging guide to help evaluate recurrent mitral valve dysfunction following repair.

29. De Backer O, Piazza N, Banai S, et al. Percutaneous transcatheter mitral valve replacement: an overview of devices in preclinical and early clinical evaluation. Circ Cardiovasc Interv. 2014;7:400-9. Contemporary reviews in the transcatheter mitral valve replacement. 
30. Feldman T, Foster E, Glower DD, et al. Percutaneous repair or surgery for mitral regurgitation. N Engl J Med. 2011;364:1395-406.

31.• Blanke P, Naoum C, Webb J, et al. Multimodality imaging in the context of transcatheter mitral valve replacement: establishing consensus among modalities and disciplines. JACC Cardiovasc Imaging. 2015;8:1191-208. Overview of currently available transcatheter mitral valve implantation.
32. Mak GJ, Blanke P, Ong K, et al. Three-dimensional echocardiography compared with computed tomography to determine mitral annulus size before transcatheter mitral valve implantation. Circ Cardiovasc Imaging. 2016;9. This study investigated the feasibility and reproducibility of D-shaped mitral annulus segmentation using 3D-transesophageal echocardiography in patients with MR. 